\title{
SEC22B Gene
}

National Cancer Institute

\section{Source}

National Cancer Institute. SEC22B Gene. NCI Thesaurus. Code C101478.

This gene is involved in protein transport. 